\title{
Literatur
}

1. Cohren, S. L. und G. F. Marrian, Biochem. J. 30, 57 (1936). 2. Colirn, S. L., G. F. Marrian und A. D. Odell, Biochem. J. 30, 2250 (1936). - 3. Corien, S. L., J. biol. Chemistry 184, 417 (1950). -4. Grant, J. K. und G. F. Marrian, Biochem. J. 47, 1 (1950). 5. Beling, C. G:, Acta endocr. (Kbh.) Suppl. 79 (1963). - 6. Carpenter, J. G. D. und A. E. Kellie, Biochem. J. 84, 303 (1962). - 7. Neeman, N. und Y. Hashimoto, J. Amer. chem. Soc. 84, 2972 (1962). - 8. Hashimoto, Y. und M. Neeman, J. biol. Chemistry 238, 1273 (1963). - 9. Slaunwhite, W. R. jr., M. A. Lichtman und A. A. Sandberg, J. Clin. Endocr. 24, 638 (1964). 10. Goebelsmann, U., K. Sjöberg, N. Wiquist und E. DiczFalusy, Acta endocr. (Kbh.) 50, 261 (1965). - 11. Diczfalusy, E. und $\mathrm{C}_{13}$. Lauritzen, Östrogene beim Menschen, Springer-Verlag,
Berlin-Göttingen-Hcidelberg (1961). - 12. DAнM, K. und H. Breuer, Acta endocr. (Kbh.), im Druck. - 13. Folin, O. und V. Ciocalteu, J. biol. Chemistry 73, 627 (1927). - 14. Brown, J. B., Biochem. J. 60, 185 (1955). - 15. NoCKE, W. Biochem. J. 78, 593 (1961). - 16. BerLINER, D. L., O. V. DomingueZ und G. Westenskow, Analytic. Chem. 29, 1797 (1957). - 17. AdLercreutz, H., Acta endocr. (Kbh.) Suppl. 72 (1962). - 18. LisBoA, B. P., I. Drossé und H. Breuer, Hoppe-Scyler's Z. physiol. Chem. 342, 106 (1965). - 19. Breuer, H., Vitamins and Hormones 20, 285 (1962). - 20. Dahm, K. und H. Breuer, Biochim. biophysica Acta (Amsterdam) 113, 404 (1966). - 21. HeCKer, E. und D. Betz, Hoppe-Seyler's Z. physiol. Chem. 338, 260 (1964).
Professor Dr. H. Breuer

Chirurgische Universitätsklinik und Poliklinik 53 Bonn-Venusberg

\section{Glykosaminoglykane und Glykosaminoglykano-Hydrolasen in Transplantationstumoren}

\author{
Von E. Buddecke und B. Sittrer \\ Aus dem Physiologisch-cbemischen Institut der Universität Tübingen (Direktor: Prof. Dr. Dr. G. Weitzel)
}

(Eingegangen am 19. Februar 1966)

Für eine Beziehung zwischen dem infiltrativen Wachstum von Tumoren und ihrem Gehalt an sauren Glykosaminoglykanen (sauren Mucopólysacchariden) und Glykosaminoglykan-hydrolysierenden Enzymen gibt es nur wenige experimentelle Belege. Das Walker-Carcino-Sarkom und Yoshida-Sarkom der Ratte und das Ebrlichsche AscitesCarcinom der Maus enthalten saure Glykosaminoglykane und Glykoproteine in einer Menge von $0,1-0,2 \%$ bzw. $0,7-1,5 \%$ bezogen auf das Trockengewicht. Die sauren Glykosaminoglykane bestehen vorwiegend aus Hyaluronat und Chondroitin 4- bzw. 6-sulfat und geringen Anteilen nicht identifizierter $z$. T. sulfat- und uronsäurehaltiger Glykosaminoglykane. Hyaluronat und Chondroitinsulfat wurden in reiner Form dargestellt und durch Analysen identifiziert. Bezüglich der untersuchten chemischen und physiko-chemischen Eigenschaften bestanden keine Unterschiede zu den sauren Glykosaminoglykanen mesenchymaler Gewebe. In 0,15-proz. Hyaluronatlösung ist die Diffusion von D-Glucose wenig, von Rinderserumalbumin stark verzögert. Die nach proteolytischem Abbau gewonnenen Glykoproteine enthalten neben einem Anteil von $50 \%$ Protein bzw. Polypeptid als Zuckerkomponenten Sialinsäure, N-Acetyl-hexosamin, D-Galaktose und D-Mannose.

Im Walker-Carcino-Sarkom sind Enzyme vorhanden, die den Abbau saurer Glykosaminoglykane bewirken. Hyaluronat-Glykanohydrolase (E.C.3.2.1.35), N-Acetyl- $\beta$-glucosaminidase (E.C.3.2.1.30) und $\beta$-Glucuronidase (E.C. 3.2.1.31) wurden in z. T. hohen spezifischen Aktivitäten nachgewiesen und durch Ammoniumsulfatfällung und Säulenchromatographie angereichert. Bei synergistischer Wirkung dieser Enzyme wird Hyaluronat bis zu monomeren Spaltprodukten abgebaut, unter denen freies N-Acetyl-glucosamin und Glucuronat isoliert und identifiziert wurden.

There is only limited experimental evidence for a relationship between the infiltrative growth of tumours and their content of acidic glycosaminoglycans (acidic mucopolysaccharides) and glycosaminoglycan-hydrolysing enzymes. On a dry weight basis, the Walker-carcino-sarcoma and the Yushida-sarcoma of the rat and the Ebrlicl Ascites-carcinoma of the mouse contain $0.1-0.2 \%$ and $0.7-1.5 \%$ respectively of acidic glycosaminoglycans and glycoproteins. The acidic glycosaminoglycans consist chiefly of hyaluronate and chondroitin 4-or 6-sulphate, with small amounts of unidentified glycosaminoglycans, which contain some sulphate and uronic acid. Hyaluronate and chondroitin sulphate were purified and identified by analysis. With regard to the chemical and physical-chemical properties studied, they showed no differences from the acidic glycosaminoglycans of mesenchymal tissue. In $0.15 \%$ hyaluronate solution, the diffusion of glucose is slighțly retarded but that of bovine serum albumin is strongly retarded. Glycoproteins, obtained by proteolytic degradation, contain $50 \%$ of protein or polypeptides, and the sugar components are sialic acid, $\mathrm{N}$-acetyl-hexosamine, $\mathrm{D}$-galactose and $\mathrm{D}$-mannose.

In the Walker-carcino-sarcoma, enzymes are present, which catalyse the degradation of acidic glycosaminoglycans. Hyaluronate-glycanohydrolase (E.C.3.2.1.35), $\mathrm{N}$-acetyl- $\beta$-glucosamidase (E.C.3.2.1.30) and $\beta$-Glucuronidase (E.C. 3.2.1.31) were found, sometimes with high specific activity, and were concentrated by ammonium sulphate precipitation and column chromatography. By the synergistic action of these enzymes, hyaluronte is degraded to monomeric cleavage products, of which free $\mathrm{N}$-acetyl-glucosamine and glucuronate were isolated and identified.

Saure Glykosaminoglykane ${ }^{1}$ ) lassen sich histochemisch

1) Vorgeschlagener systematischer Name für aminozuckerhaltige Polysaccharide, die pro Disaccharideinheit eine Uronsäure und/oder eine oder mehrere Estersulfatgruppen enthalten. Siehe auch: M. FLORKIN u. E. H. Stotz, Comprehensive Biochemistry, Vol. 5, Carbohydrates, S. 262ff; Elsevier Publishing Comp. Amsterdam, London, New York, (1963). in vielen gut- und bösartigenTumoren epithelialer und mesenchymaler Herkunft nachweisen (1). Ihre Akkumulation an der Grenze zwischen Tumor- und Wirtsgewebe steht mit der Frage einer möglichen Funktion der Tumorglykosaminoglykane als „Permeabilitätsbarriere“ bzw. einer ,,mesenchymalen Abwehrreaktion“ des W/irts- 
organismus in engem Zusammenhang. Eine Prüfung dieser Frage setzt jedoch Kenntnisse über die chemische Struktur und den Stoffwechsel der Tumorglykosaminoglykane voraus. Hyaluronat und Heparin wurden zwar in verschiedenen Tumoren mit chemisch-analytischen Methoden nachgewiesen, doch beschränken sich die Angaben bei Tiertumoren auf das Rous-Sarkom beim Huhn (2), das Ebrliclssche Ascites Carcinom (3) und den Mastzelltumor (4) bei der Maus. Zudem wurde eine Reindarstellung und chemische Charakterisierung meist nicht versucht.

Im folgenden wird über die Isolierung und chemische Identifizierung saurer Glykosaminoglykane aus Transplantationstumoren der Ratte (Walker-Carcino-Sarkom, Yosbida-Sarkom) und dem Ebrlicbschen Ascites-Carcinom der Maus und ihren Vergleich mit sauren Glykosaminoglykanen mesenchymaler Gewebe berichtet. Die Auswahl des Untersuchungsgutes erfolgte unter dem Gesichtspunkt, daß über den Gehalt an Glykosaminoglykanen der beiden in der tierexperimentellen Tumorforschung in ausgehendem Umfange eingesetzten Rattentumoren noch keine Angaben vorliegen. Sowohl für den Stoffwechsel der Tumorglykosaminoglykane als auch für die Fähigkeit der Tumoren zu infiltrativem Wachstum in das Nachbargewebe ist das Wirksamwerden glykosaminoglykan-abbauender Enzyme notwendig. Dieser Frage wurde durch Nachweis, Anreicherung, Aktivitätsbestimmung und Untersuchung der Wirkungsweise von drei verschiedenen synergistisch wirkenden Glykosaminoglykanohydrolasen nachgegangen.

\section{Methodik}

\section{Untersucbungsgut}

Auf Wistarratten transplantierte Walker- und Yoshidatumoren mit einem Gewicht von $10-30 \mathrm{~g}$ werden von Fremdgewebe und Nekrosen freipräpariert und zur Isolierung der sauren Glykosaminoglykane unter mechanischer Zerkleinerung in Acetontrockengewebe überführt. Bei Enzymaufarbeitungen wurde Tumorfrischgewebe wie unten beschrieben, weiterverarbeitet.

Ebrlichsches Mäuseascites-Carcinom wurde mittels Punktion am 11. Tage nach der Transplantation gewonnen. Bei getrennter Aufarbeitung von Zellen und Serum wurden die abzentrifugierten Zellen zweimal mit kalter Ringerlösung gewaschen, Serum und Waschwasser und die gewaschenen Zellen getrennt - im anderen Falle die gesamte Ascitesflüssigkcit - in Acetontrockenmaterial überführt bzw. in Citratpuffer homogenisiert.

Lyophylisiertes Material aus der Pleuraflüssigkeit eines lymphangiomähnlichen Mesotbelioms wurde in dankenswerterweise durch Prof. E. LANGer, Pathologisches Institut, München-Schwabing, zur Verfügung gestellt.

\section{Analysen}

Die Bestimmung der Gesamthexosamine, der Nichtaminozucker, der Neuraminsäure, der Uronsäure und L-Fucose sowie die getrennte Bestimmung von Glucosamin und ${ }^{-G a l a k t o s a m i n ~ w u r d e ~}$ - wie früher beschricben (5) - durchgeführt. Die Identifizierung der Neutralzucker erfolgte. durch Papierchromatographie in n-Butanol/Pyridin/Wasser (8:6:3) nach Hydrolyse mit Dowex 50 in der $\mathrm{H}+$ Form. Die Bestimmung der reduzierenden Endgruppen (N-Acetyl-hexosaminäquivalente) in Hyaluronat bzw. Chondroitin 6-sulfat nach enzymatischem Abbau wurde nach 1. c. (6) vorgenommen.

\section{Präparative Darstellung der sauren Glykosaminoglykane}

Für die Reindarstellung der im Tumorgewebe enthaltenen Glykosaminoglykane aus Acetontrockengewebe wurden zunächst die Gesamtglykosaminoglykane nach Proteolyse mit Papain und Äthanolfällungen bei verschiedenen $\mathrm{pH}$ isoliert. Die Trennung von sauren Glykosaminoglykanen und Glykoproteinen erfolgte durch Phenolextraktion, die Fraktionierung der sauren Glykosaminoglykane über ihre Cetylpyridiniumkomplexe bzw. durch Chromatographie an ECTEOLA-Cellulose, wie in 1. c. (5) beschrieben.

\section{Enzymanreicherung und Aktivitätsmessungen}

Durch saubere Präparation isoliertes Tumorgewebe aus WalkerCarcino-Sarkom wurde sofort mit dem fünffachen Vol. eiskaltem 0,1 м Citratpuffer mit $0,15 \mathrm{M} \mathrm{NaCl}$ (pH 4,5) $2 \mathrm{Min}$. im Ultraturrax unter Kühlung im Eisbad homogenisiert und $24 \mathrm{Stdn}$. bei $-20^{\circ}$ gehalten. Nach Wiederauftauen und $30 \mathrm{Min}$. Rühren bei $+4^{\circ}$ wurde bei $78000 \times g$ abzentrifugiert und der Uberstand einer fraktionierten Ammoniumsulfatfällung unterworfen. Die bei Ammoniumsulfatkonzentrationen von $0-10,11-30$ und $31-40 \%$ gewonnenen Niederschläge wurden in destilliertem Wasser aufgenommen, zweimal 24 Stdn. gegen destilliertes Wasser dialysiert und lyophylisiert. Die höchssten Aktivitäten der geprüften Enzyme wurden in der Fraktion von 11-30\% Ammoniumsulfat gefunden. 0,055 g der Fraktion 11--30 wurden auf eine mit Sephadex G 75 beschickte und mit 0,05 M Phosphatcitratpuffer mit 0,15 M Kochsalz $(\mathrm{pH} \mathrm{4,1)}$ äquilibrierte Säule $(0,5 \times 70 \mathrm{~cm})$ gegeben und mit dem gleichen Puffer eluiert. Das Eluat wurde kontinuierlich über einen Kapillarschlauch auf eine zweite mit Sephadex G 100 gefüllte und in gleicher Weise äquilibrierte Chromatographiesäule gleichen Volumens gegeben und das zweite Eluat in Einzelfraktionen von je $1 \mathrm{ml}$ aufgefangen. Der Proteingehalt wurde durch kontinuierliche Messung bei $254 \mathrm{~m} \mu$ registriert, über einen Punktschreiber aufgezeichnet und in den einzelnen Fraktionen durch Messung der Extinktion bei $280 \mathrm{~m} \mu$ kontrolliert.

Die Aktivitätsmessungen erfolgten für Hyaluronatglykanohydrolase (E.C. 3.2.1.35) nach 1. c. (7) bei pH 4,1 unter Verwendung von Hyaluronat als Substrat, für $\mathrm{N}$-Acetyl- $\beta$-Glucosaminidase (E.C. 3. 2. 1.30) bei $\mathrm{pH} 4,5$ mit Phenyl- $\beta$-N-acetylglucosaminid nach 1. c. (8) und für $\beta$-Glucuronidase (E.C. 3. 2. 1. 31) bei $\mathrm{pH} 5,2$ nach 1.c. (9) mit Phenolphthalein- $\beta$-Glucuronid als Substrat. Bestimmung der proteolytischen Aktivität bei pH 3,1 mit denaturiertem Hämoglobin als Substrat nach 1. c. (10).

\section{Ergebnisse}

Isòlierung und chemische Zusammensetzung saurer Gly'kosaminoglykane und Glykoproteine

Der Gehalt an Aminozuckern liegt im Acetontrockengewebe solider Transplantationstumoren mit $0,5-1 \%$, derjenige von Hydroxyprolin mit $0,8 \%$ um eine Gößenordnung niedriger als in polysaccharidreichen mesenchymalen Geweben. Infolge des vorwiegend zellulären Aufbaues tritt also der Anteil an extrazellulären Polysacchariden und des Faserproteins Kollagen im Tumorgewebe zwar stark zurück, ist bei den hier untersuchten soliden Tumoren jedoch ausnahmslos vorhanden und demnach ein charakteristischer Bestandteil. Die präparative Darstellung der Gesamtglykosaminoglykane (Summe der sauren Glykosaminoglykane und Glykoproteine) ergab einen Gehalt von $2 \%$ für das Walker-CarcinoSarkom, 0,9\% für das Yoshida-Sarkom, 1,7\% für das Ebrlichsche Ascitescarcinom, wobei jedoch, wie Tabelle 1 zeigt, die sauren Glykosaminoglykane nur mit 0,06 bis $0,15 \%$ beteiligt, die Glykoproteine dagegen in einer zehnmal größeren Menge im Tumorgewebe vorhanden sind (s. Tab. 2). Bei dieser Angabe ist zu berücksichtigen, $\mathrm{da} ß$ es sich bei den Glykoproteinen lediglich um die 
Tab. 1.

Zusammensetzung von K-Hyaluronat und K-Chondroitinsulfat aus Transplantationstumoren und einem Human-Mesotheliom Angaben für GlcN, GalN, GUA, N und S in \% der Reinsubstanz. GlcN = Glucosamin, GalN = Galaktosamin, GUA = Glucuronsäure

\begin{tabular}{|c|c|c|c|c|c|c|c|c|c|c|c|}
\hline \multirow[b]{2}{*}{ Tumor } & \multicolumn{5}{|c|}{ K-Hyaluronat } & \multicolumn{6}{|c|}{ K-Chondroitinsulfat } \\
\hline & $\begin{array}{l}\text { Gehalt } \\
\text { in \% TG }\end{array}$ & GlcN & $\stackrel{\%}{\text { GUA }}$ & $\mathbf{N}$ & {$[\alpha]_{\nu}^{20}$} & $\begin{array}{l}\text { Gehalt } \\
\text { in \% TG }\end{array}$ & GalN & GUA $^{\%}$ & $\mathrm{~N}$ & $\mathrm{~S}$ & $\begin{array}{c}\text { IR-Bande } \\
\left(\mathrm{cm}^{-1}\right)\end{array}$ \\
\hline $\begin{array}{l}\text { Walker-Carcino- } \\
\text { Sarkom }\end{array}$ & 0,050 & 32,2 & 37,2 & 3,9 & $-19,5$ & 0,10 & 24,5 & 23,2 & 4,7 & 4,8 & 850 \\
\hline Yosbida-Sarkom & 0,013 & 22,8 & 20,5 & 5,4 & $-2,3$ & 0,05 & 20,9 & 16,4 & - & 4,73 & 855 \\
\hline $\begin{array}{l}\text { Ebrlicb-Ascitcs- } \\
\text { Carcinom (total) }\end{array}$ & 0,036 & 23,4 & 21,5 & 4,8 & $-21,0$ & 0,018 & 14,5 & 14,1 & 5,2 & - & $\begin{array}{c}1000,815 \\
860\end{array}$ \\
\hline $\begin{array}{l}\text { Ebrlicb-Ascites- } \\
\text { Carcinom (Serum) }\end{array}$ & 0,017 & 38,9 & 44,8 & 2,7 & $-46,4$ & & & & & & \\
\hline Mesotheliom & 9,0 & 40,0 & 47,6 & 2,6 & $-56,0$ & & & & & & \\
\hline
\end{tabular}

Tab. 2.

Chemische Zusammensetzung phenollöslicher Glykoproteine aus Transplantationstumoren; Angaben in \% der Trockensubstanz

\begin{tabular}{lccccccc}
\hline & $\begin{array}{c}\text { Hexosamin } \\
\text { total }\end{array}$ & $\begin{array}{c}\text { Neutralzucker } \\
\text { (Orcin) }\end{array}$ & $\begin{array}{c}\text { Relation Fucose: } \\
\text { Mannose: Galaktose }\end{array}$ & Fucose & Sialinsäure & $\begin{array}{c}\text { Stickstoff } \\
\text { Gehalt in } \\
\% \text { des TG }\end{array}$ \\
\hline $\begin{array}{lcccccc}\text { Walker-Carcino- } \\
\quad \text { Sarkom 258 }\end{array}$ & 13,9 & 6,9 & $1: 3: 5$ & 0,72 & 12,0 & 7,72 & 1,40 \\
$\begin{array}{l}\text { Yoshida-Sarkom } \\
\text { Ebrlich-Ascites-Carcinom }\end{array}$ & 19,2 & 9,5 & $1: 3: 5$ & 0,82 & 23,4 & 5,95 & 0,74 \\
\hline
\end{tabular}

nach Proteolyse zurückbleibenden nicht dialysierbaren und phenollöslichen Anteile der ursprünglich im Tumorgewebe enthaltenen Gesamtglykoproteine handelt (s. Methodik). Die Fraktionierung und Reindarstellung chemisch definierter saurer Glykosaminoglykane, die über die Cetylpyridiniumkomplexe sowie durch Ionenaustauschchromatographie durchgeführt wurde, und deren Ergebnisse ebenfalls in Tabelle 1 dargestellt sind, ergab mit Ausnahme des Mesothelioms für alle Tumoren die Anwesenheit mehrerer saurer Glykosaminoglykantypen und führte zur Isolierung von hochgereinigtem Hyaluronat, Chondroitin 4-sulfat und Chondroitin 6-sulfat.

Bei den Hyaluronsäurepräparaten der Transplantationstumoren wurden trotz der erreichten guten molaren Proportionalität zwischen Hexosamin- und Uronsäurewert $\mathrm{zu}$ hohe Stickstoffwerte und zu niedrige Werte des Absolutgehaltes und der optischen Drehung gefunden. Diese Abweichungen sind auf die Anwesenheit von Ribonucleinsäuren zurückzuführen, die zwar bei der Cetylpyridiniumfraktionierung weitgehend aber nicht vollständig entfernt werden konnten. Auch nach wiederholter Chromatographie der gereinigten sauren Glykosaminoglykane, die aus zellulärem Ausgangsmaterial erhalten wurden, ließen sich noch Spuren von Adenin, Guanin, Phosphat und Ribose sowie ein Absorptionsmaximum bei $258 \mathrm{~m} \mu$ nachweisen. Dagegen lieferte die Isolierung von Hyaluronat aus zellfreien Medien (Ascitesserum, Mesotheliomflüssigkeit) Präparate von hoher Reinheit.

Auch bei der Isolierung von Chondroitinsulfat, das in Walker- und Yosbida-Tumoren in größeren, in Ascites Tumoren in geringeren Mengen vorhanden ist als Hyaluronat, bereitete die Abtrennung von Nucleinsäure
Schwierigkeiten. Das beste Präparat wurde aus WalkerCarcino-Sarkom gewonnen. Bei der Differenzierung der Chondroitinsulfat-Isomeren durch das IR-Spektrum ließ das aus Walker - und Yosbidatumoren isolierte Präparat lediglich die für eine axiale Position der Estersulfatgruppe charakteristische Bande bei $850-855 \mathrm{~cm}^{-1}$ erkennen, so daß eine Zuordnung zum Chondroitin 4-sulfat möglich war. Der etwas zu geringe Uronsäuregehalt im Chondroitpräparat des Yoshida-Sarkoms könnte jedoch durch die Anwesenheit von Iduronsäure bedingt sein, so daß hier möglicherweise als zweiter Chondroitinsulfattyp Dermatansulfat vorliegt. Beim Ascites-Chondroitinsulfat war im IR-Spektrum zusätzlich die für C-6-Substitution des Sulfatesters typische Schwingung bei 1000 und $815 \mathrm{~cm}^{-1}$ erkennbar, was auf das Vorhandensein von Chondroitin 4- und 6-sulfat schließen läßt, doch ist bei dem geringeren Reinheitsgrad die Beimischung weiterer 6-Sulfatester enthaltender Polysaccharide nicht auszuschließen.

Chondroitinsulfat und Hyaluronat machen zusammen etwa 60-80\% der gesamten sauren Glykosaminoglykane aus. Daneben wurden mit Ausnahme des Mesotbelioms in allen Transplantationstumoren weitere sulfatierte saure Glykosaminoglykane nachgewiesen, die jedoch wegen ihrer geringen Menge und ihres unzureichenden Reinheitsgrades nicht identifiziert werden konnten. Bei einer getrennten Analyse zellulärer und extrazellulärer $\dot{\mathrm{Ge}}$ webeanteile, die bei den soliden Tumoren nach Trypsinierung vorgenommen wurde, zeigte sich erwartungsgemä $\beta$, daß der überwiegende Anteil der sauren Glykosaminoglykane (beim Ebrlicbschen Ascites-Carcinom 75\%) extrazellulär lokalisiert ist. Bei einem Vergleich der chemischen Zusammensetzung und der physiko-chemischen Eigenschaften der aus den Tumoren isolierten 
sauren Glykosaminoglykane mit authentischem Hyaluronat bzw. Chondroitinsulfatpräparaten ergaben sich keine Unterschiede. Beim Abbau mit Testeshyaluronidase (E. C. 3. 2. 1.35) wurden die Hyaluronatfraktionen während 30 Min. mit gleicher (Mesotheliom) oder nur geringfügig reduzierter, die Chondroitinsulfate jedoch z. T. mit bis auf $50 \%$ herabgesetzter Reaktionsgeschwindigkeit gespalten.

$\mathrm{Da}$ - wie früher beobachtet (11) - makromolekulare Chondroitinsulfat-Proteine einen Molekularsiebeffekt entfalten, wurde auch für das hochvisköse Kaliumhyaluronat aus menschlichem Mesotheliom $([\eta]=2,6)$ geprüft, ob es als selektives Filter wirksam werden könnte. Hierzu wurden einmal der Gasstoffwechsel von Asciteszellen in Gegenwart von Hyaluronat $(4 \mathrm{mg} / \mathrm{ml})$ untersucht, zum anderen in Modellversuchen mit der Diffusionskammer (11) das Diffusionsverhalten verschiedener Moleküle bei der Passage von Hyaluronatlösungen verschiedener Konzentrationen geprüft. Während die Warburg-Versuche ergaben, daß endogene und Gluçose-Atmung von Asciteszellen sowie anaerobe Glykolyse durch Hyaluronat nicht beeinflußt werden, wurde in Versuchen mit der Diffusionskammer das in Abbildung 1 dargestellte Ergebnis erhalten. Während Glucose und Histidyl-Histidin Hyaluronatlösungen einer Konzentration von $5-15 \mathrm{mg} / \mathrm{m} l$ nahezu ungehindert passieren, wird für Rinderserum-Albumin mit zunehmender Hyaluronatkonzentration eine steigende Diffusionshemmung beobachtet. Hochmolekulares Hyaluronat aus Nabelschnur, das in Kontrollversuchen geprüft wurde, lieferte gleiche Ergebnisse.

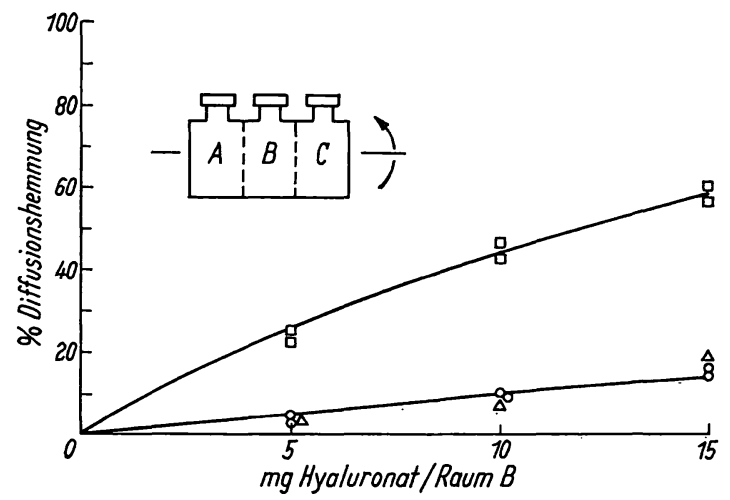

Abb. 1

Diffusionshemmung für D-Glucose $(O)$, L-Histidyl-Histidin $(\triangle)$ und Rinderserumalbumin $(\square)$ bei der Penetration durch Hyaluronatlösungen in der Versuchsanordnung nach 1.c. (11). Bei Versuchsbeginn enthält die Kammer A Glucose $(1,5 \mathrm{mg} / \mathrm{m} l)$, HistidylHistidin $(4 \mathrm{mg} / \mathrm{m} /)$ bzw. Albumin $(10 \mathrm{mg} / \mathrm{m} l)$, die Kammer B Hyaluronat gelöst in 0,1 M Acetatpuffer $\mathrm{pH} 6$, Kammer C Puffer. Trennung der Kammern durch Cellophanmembranen bzw. Millipore-Filter (Porendurchmesser $1000 \AA$ )

Bei den aus Transplantationstumoren isolierten Glykoproteinen ist eine Klassifizierung in einzelne Typen nicht möglich. Die Aussage, daß der überwiegende Teil der Gruppe der sialinsäurehaltigen Glykoproteine angehört, läßt sich jedoch trotz der proteolytischen Vorbehandlung des Aufarbeitungsansatzes treffen, da wegen der protektiven Wirkung der Sialinsäure bei der Proteolyse von Glykoproteinen (12) zumindest bezüglich der kohlenhydrathaltigen prosthetischen Gruppe mit großer Wahrscheinlichkeit ein repräsentativer Anteil höhermolekularer Glykoproteinspaltprodukte isoliert wurde. Ihr hoher Sialinsäuregehalt ist im Hinblick auf den im Vergleich zum benachbarten Normalgewebe erhöhten Sialinsäuregehalt im Tumorgewebe (13) und die Isolierung sialinsäurehaltiger Glykopeptide aus Ebrlichschen Ascites-Carcinomzellen (14) von Interesse.

Aus diesen Ergebnissen kann geschlossen werden, daß die Transplantationstumoren von Ratte und Maus über ein spezifisches Spektrum von mindestens zwei - wahrscheinlich jedoch mehr - Typen von sauren Glykosaminoglykanen aufweisen. Lediglich der Tumor mesenchymaler Herkunft produziert nur ein einziges - der Synthesefähigkeit der Stammzelle entsprechendes saures Glykosaminoglykan.

\section{Glykosaminoglykano-Hydrolasen in Transplantationstumoren}

Hohe Umsatzraten saurer Glykosaminoglykane, die für mesenchymale Gewebe mit biologischen Halbwertzeiten in einer Größenordnung von 3-14 Tagen liegen, bedingen zeitliche Korrespondenz und vergleichbare Geschwindigkeit biosynthetischer und kataboler Stoffwechselprozesse. $\mathrm{Da}$ einerseits für Transplantationstumoren derartige Untersuchungen bisher nicht vorliegen, andererseits die beim Abbau der sauren Glykosaminoglykane beteiligten Enzyme im Arteriengewebe $(7,8)$ näher charakterisiert wurden, wựde die Existenz und Wirkungsweise Glykosaminoglykan-abbauender Enzyme auch im Walker-Carcino-Sarkom untersucht.

Für den Abbau von Hyaluronat und Chondroitinsulfat bis zu ihren monomeren Bausteinen ist die synergistische bzw. alternierende Wirkung mehreter Hydrolasen erforderlich. Beim enzymatischen Abbau des Hyaluronats folgt der depolymerisierenden Wirkung einer Hyaluronatglykanohydrolase (E. C. 3. 2. 1. 35) die schrittweise Verkürzung der entstandenen Hyaluronatoligosaccharide dürch die Exoenzyme $\beta$-Glucuronidase (E. C. 1. 2. 1. 31) bzw. N-Acetyl- $\beta$-hexosaminidase (E. C. 3. 2. 1. 30) vom nichtreduzierenden Ende her. Das Zusammenwirken dieser Enzyme im Tumorgewebe ist nicht nur im Hinblick auf den Stoffwechsel der sauren Glykosaminoglykane von Interesse, sondern bildet möglicherweise auch die Voraussetzung für das infiltrative Wachstum des Tumors und der damit verbundenen Notwendigkeit, die sauren Glykosaminoglykane der Extrazellulärsubstanz des Nachbargewebes enzymatisch aufzulösen. Da Chondroitinsulfat im Nativzustand als Proteinkomplex vorliegt, ist möglicherweise auch die Mitwirkung von Proteasen und Peptidasen von Bedeutung.

Aus Tabelle 3 geht hervor, daß alle diese Enzyme im Tumorgewebe des Walker-Carcino-Sarkoms vorhanden sind und z. B. für N-Acetyl- $\beta$-hexosaminidase und $\beta$ Glucuronidase Aktivitäten nachweisbar sind, welche diejenige anderer Organe des Wirtstieres $z$. T. um ein mehrfaches übertreffen. Bei der Angabe von Aktivitäten für die Hyaluronatglykanohydrolase ist zu berücksichtigen, daß einmal die Existenz dieses Enzyms außerhalb des Tumorgewebes überhaupt als problematisch galt, 
Tab. 3.

Spezifische Aktivität von Glykosaminoglykano-Hydrolasen und protein- bzw. peptidspaltenden Enzymen in verschiedenen Organen der Ratte; Aktivitätsmessungen unter optimalen Bedingungen

\begin{tabular}{lcccccc}
\hline Organ & $\begin{array}{c}\text { Protcin im Uber- } \\
\text { stand des Homo- } \\
\text { genates } \mathrm{mg} / \mathrm{ml}\end{array}$ & Hyaluronidase & $\beta$-Glucuronidase & $\begin{array}{c}\text { Spezifische Aktivität in mU } \\
\text { N-Ac-Glucos- } \\
\text { aminidase }\end{array}$ & $\begin{array}{c}\text { Kathepsin } \\
\text { saure Carb- } \\
\text { oxypcptidase') }\end{array}$ \\
\hline Walker-Carcino-Sarkom & 6,6 & 0,346 & 24,2 & 91,2 & 1,3 & 11,3 \\
Leber & 16,0 & 0,129 & 9,2 & 35,1 & 2,1 & 5,4 \\
Niere & 9,3 & 0,109 & 2,3 & 103,0 & 4,8 & 12,2 \\
Muskel & 7,3 & 0,057 & 1,9 & 12,7 & 0,9 & 0,5 \\
Milz & 14,1 & & 11,9 & 22,4 & 6,2 & 6,9 \\
\hline
\end{tabular}

1) Die saure Carboxypeptidase ist ein bisher unbekanntes Enzym, das bei einem pH-Optimum von 4,5-4,6 von Tri-(und 'Tetra-)peptiden die C-terminale Aminosäure abspaltet. Die Spezifität der sauren Carboxypeptidase erstreckt sich - soweit bisher untersucht - vorzugsweise auf terminale Leucyl-, Valyl- und Phenylalanyl-Gruppen. Von der Pankreas-Carboxypeptidase unterscheidet sich dic auch in anderen Geweben vorhandene saure Carboxypeptidase durch das pH-Optimum und durch Wirkungslosigkeit gegenüber dem synthetischen Substrat N-Cbo-Glycyl-L-phenylalanin. Der Aktivitätstest für die saure Carboxypeptidase erfolgte unter Verivendung von L-Seryl-Ileucyl-L-leucin als Substrat in 0,05 M Citratphosphatpuffer pH 4,5 mit nachfolgender Bestimmung des freigesetzten Leucins durch die Ninhydrin-Reaktion in der Modifikation nach (15).

und zum anderen beim Aktivitätstest die gleichzeitige Anwesenheit von $\beta$-Glucuronidase und $\mathrm{N}$-Acetyl- $\beta$ hexosaminidase interferiert und $\mathrm{zu}$ hohe Werte vorgetäuscht werden. Nach den hier gewonnenen Ergebnissen steht die Existenz einer Hyaluronatglykanohydrolase jedoch außer Zweifel, da die alleinige Einwirkung von $\beta$-Glucuronidase und $\mathrm{N}$-Acetyl $\beta$-hexosaminidase auf polymere Hyaluronsäure keine Freisetzung der hier im Aktivitätstest erfaßten reduzierenden N-Acetylglucosaminendgruppen bewirkt.

Eine Ammoniumsulfatfraktionierung des Tumorhomogenatüberstandes und deren Chromatographie an Sephadex G 75 und $G 100$ führt zu teilweiser Trennung und Anreicherung der Enzymaktivitäten. Abbildung 2 gibt das Elutionsdiagramm der Ammoniumsulfatfraktion 11-30 nach Dextrangelfiltration wieder, dem zu entnehmen ist, daß trotz der erreichten Proteinfraktionie-

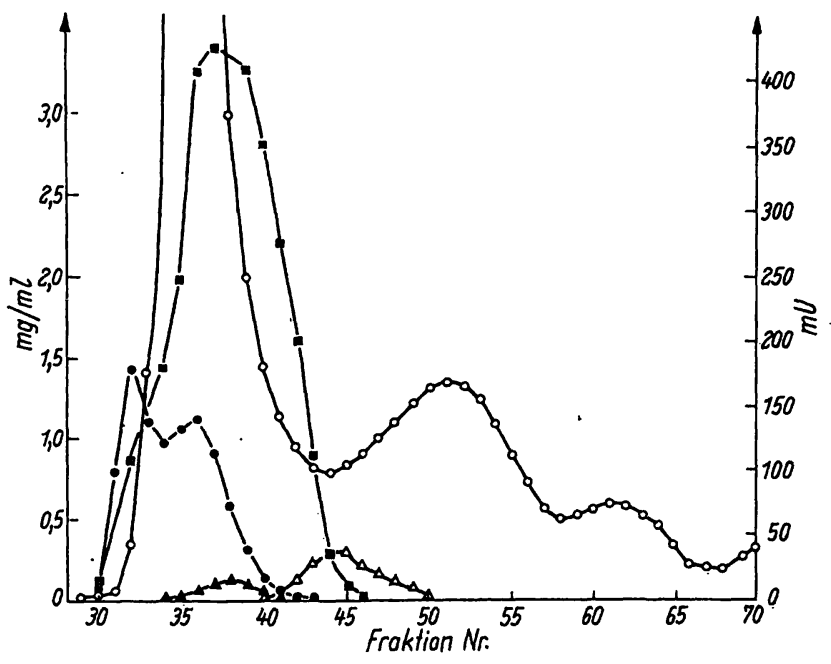

Abb. 2

Verteilung der Enzymaktivitäten nach säulenchromatographischer Fraktionierung (Sephadex G 75 und G 100) der durch Ammoniumsulfatfällung (11-30 Gew.\%) gewonnenen Proteinfraktion aus Homogenat von Walker-Carcino-Sarkom.

Linke Ordinate: $\mathrm{mg}$ Protein $/ \mathrm{m} /$; Recbte Ordinate: spezifische Enzymaktivitäten in $\mathrm{mU}$. $\bullet=\beta$-Glucuronidase, $\|=\mathrm{N}$-Acetyl$\beta$-glucosaminidase, $\Delta=$ Hyaluronat-Glukanohydrolase (Maßstab $1: 25$ vergrößert), $\Delta=$ Protease rung die Tumorglycosidasen ausnahmslos im ersten Gipfel, d. h. in einer Protein mit einem Mol.-Gew. von mehr als 50000 enthaltenden Fraktion lokalisiert sind und nur die Protease abgetrennt wurde. Der weitgehenden Aufschlüsselung des Proteinspektrums mit Abtrennung von Nichtenzymprotein entspricht dic Anreicherung der einzelnen Enzyme, die für $\mathrm{N}$-Acetyl- $\beta$ hexosaminidase, $\beta$-Glucuronidase und Proteinase etwa das zehnfache der spezifischen Aktivität des Homogenates beträgt. Die Reinigung der Hyaluronatglykanohydrolase scheint mit einem Anreicherungsfaktor von 2 weniger effektvoll, doch ist $z u$ berücksichtigen, $d a ß$ ein großer Anteil der $\beta$-Glucuronidaseaktivität von der Hyaluronatglykanohydrolaseaktivität abgetrennt wurde und dadurch die durch $\beta$-Glucuronidase und $\mathrm{N}$-Acetyl$\beta$-hexosaminidase bedingten Additionseffekte abgeschwächt werden. Der errechnete Anreicherungsgrad ist daher ein Mindestwert. Die hohen Aktivitäten von NAcetyl- $\beta$-hexosaminidase und $\beta$-Glucuronidase im Tumorgewebe stehen in eigenartigem Kontrast zu ihrer bisherigen noch unbekannten physiologischen Bedeutung. Angesichts der nur geringen Konzentrationen an Hyaluronat und Chondroitinsulfat im Tumorgewebe ist zu vermuten, daß sich ihre Wirkung nicht ausschließßlich auf den Stoffumsatz der sauren Glykosaminoglykane des Tumors, sondern möglicherwreise auch auf die sauren Glykosaminoglykane des Wirtsgewebes erstreckt.

$\mathrm{Da}$ das pH-Optimum aller Tumorglykosidhydrolasen zwischen $\mathrm{pH} 4$ und 5 liegt, ist eine simultane und synergistische Wirkung an polymeren Substraten möglich. In Versuchen, bei denen die im Gipfel 1 der Abbildung 2 nachgewiesenen Glykosidasen nach Konzentrierung durch Druckfiltration zusammen auf Kaliumhyaluronat über 48 Stdn. einwirkten, konnten die enzymatisch freigesetzten monomeren Bausteine der Hyaluronsäure durch Säulenchromatographie isoliert und identifiziert werden. Abbildung 3 zeigt das Ergebnis der Chromatographie des Inkubationsansatzes an Sephadex G 25, in dem neben Oligosacchariden des Hyaluronates (Fraktion-Nr. 14-22) freies N-Acetylglucosamin und freie Glucuronsäure (Fraktion-Nr. 22-32) nachgewiesen wur- 


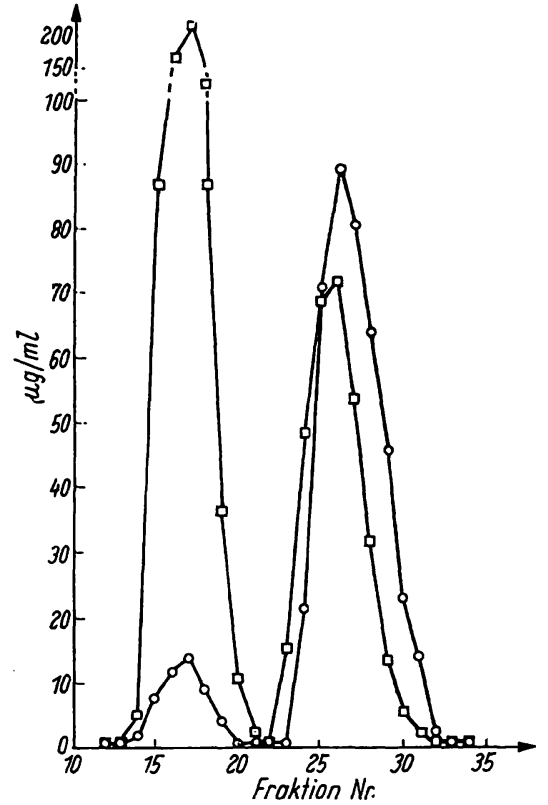

Abb. 3

Chromatographische Trennung von Hyaluronat-Oligosacchariden und freiem GNAc bzw. freier Glucuronsäure durch Gelfiltration (Sephadex G 25) aus $10 \mathrm{mg}$ Kaliumhyaluronat nach simultaner Inkubation mit Hyaluronat-Glukanohydrolase, $\beta$-Glucuronidase und $\mathrm{N}$-Acetylglucosaminidase (Fraktion $30-42 \operatorname{der} \mathrm{Abb} .2$ ) aus Walker-Carcino-Sarkom.

$\square=$ freje und glykosidisch gebundene Glucuronsäure (Carbazolreaktion)

$\mathrm{O}=$ reduzierendes GNAc (Elson-Morgan-Reaktion)

den. Der unterschiedliche Ausfall, der freies bzw. reduzierendes N-Acetyl-glucosamin erfassenden ElsonMorgan-Reaktion und der Reaktion auf freie und glykosidisch gebundene Glucuronsäure (Carbazol-Reaktion) zeigt die Anwesenheit von Hyaluronatoligosacchariden im ersten Gipfel an, bei denen das molare Verhältnis von reduzierendem N-Acetyl-hexosamin und Gesamtglucuronsäure klein ist. Dagegen lassen sich im zweiten Gipfel (Fraktion-Nr. 22-32) in äquimolarem Mengenverhältnis freie Glucuronsäure und freies N-Acetylglucosamin nachweisen, die beide als Endprodukte des enzymatischen Abbaues anfallen und papierchromatographisch identifiziert werden konnten.

Auch Chondroitinsulfat ist Substrat der Tumorglykosidasen des Walker-Carcino-Sarkoms. Wie die mit der gleichen Enzymkombination aufgenommene Zeitumsatzkurve (Abb. 4) ergibt, wird auch Chondroitin 6-sulfat - wenn auch in bedeutend geringerem Ausma $B$ als Hyaluronat - gespalten, was sich an der Zunahme der reduzierenden $\mathrm{N}$-Acetyl-hexosaminendgruppen zeigt. Die Abnahme der Reaktionsgeschwindigkeit mit der Zeit hängt damit zusammen, daß die Freisetzung reduzierender N-Acetyl-glucosaminendgruppen hier eine Resultante drei verschiedener enzymatischer Reaktionen ist und ferner mit suboptimalen Substratkonzentrationen (Begrenzung der Löslichkeit des Hyaluronats) gearbeitet wurde.

Aus den Ergebnissen geht hervor, daß im WalkerCarcino-Sarkom alle Enzyme vorhanden sind, die einen vollständigen Abbau des polymeren Hyaluronats bis $\mathrm{zu}$ den monomeren Bestandteilen ermöglichen.

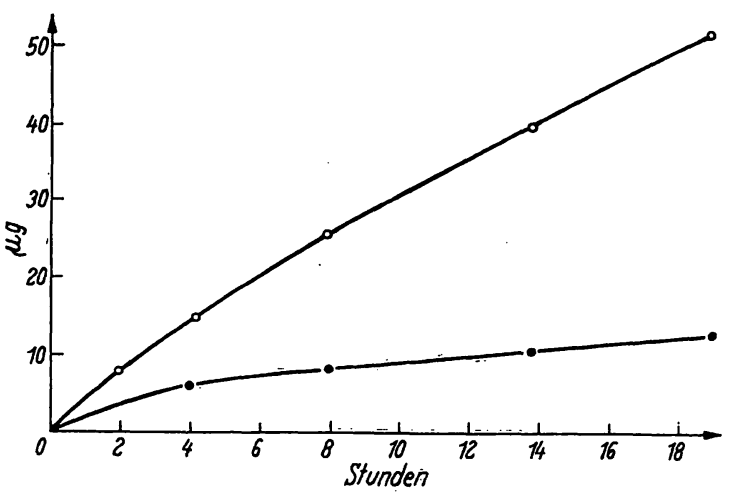

Abb. 4

Freisetzung von reduzierenden GNAc- bzw. GalNAc-Endgruppen aus Hyaluronat (O) bzw. Chondroitin 6-sulfat (o) durch die in der Ammoniumsulfat-Fraktion 11-30 enthaltenen Enzyme aus Walker-Carcino.Sarkom in Abhängigkeit von der Zeir

\section{Diskussion}

Die regelmäßige Existenz saurer Glykosaminoglykane (saurer Mucopolysaccharide) im Tumorgewebe, ihre intra- und extrazelluläre Lokalisation und ihre Akkumulation in der infiltrativ-destruierend in das Nachbargewebe vordringenden Randzone (1) legen die Vermutung nahe, daß Mucopolysaccharid-Synthese und Tumorwachstum gekoppelte Vorgänge sind. Allerdings läßt sich die Frage nach der Herkunft der sauren Glykosaminoglykane im .Tumorgewebe nicht definitiv entscheiden, da nach histochemischen Untersuchungen sowohl die Tumorzelle selbst als auch die Fibroblasten des bindegewebigen Stromas des Tumorgewebes als Produzenten der sauren Mucopolysaccharide in Betracht kommen. Das Problem wird weiterhin dadurch kompliziert, daß auch normales rasch wachsendes Gewebe (Embryonalgewebe, Granulationsgewebe) einen erhöhten Gehalt an sauren Glykosaminoglykanen aufweist (16), was auf eine ganz allgemeine Beziehung zwischen Wachstum und Mucopolysaccharidgehalt eines Gewebes hindeutet und zur Theorie einer generellen regulativen Funktion der sauren Glykosaminoglykane bzw. ihrer Calciumkomplexe bei der Zellteilung geführt hat (17).

Eine ganz andere Bedeutung der sauren Glykosaminoglykane könnte in ihrer Funktion als „Permeabilitätsbarriere" liegen, deren Effekt $z$. B. an einer Verhinderung des Eindringens von Makromolekülen aus dem Wirtsorganismus in den Tumor bei ungehinderter Passage niedermolekularer Substrate des Tumorstoffwechsels (Glucose, Aminosäuren, Peptide) bestehen könnte. Der vorstehend beschriebene „Molekularsiebeffekt" des Mesotheliom-Hyaluronats ist mit einer solchen Annahme vereinbar.

Unter den hier im Walker-Carcino-Sarkom nachgewiesenen Glykosaminoglykano-Hydrolasen war die $\beta$-Glucuronidase und ihre hohe Aktivität schon bekannt (18), doch macht erst die Existenz der weiteren vorstehend beschriebenen Glykosidasen ihre mögliche Funktion verständlich, für die sich zwei grundlegende Aspekte ergeben: einmal ist eine Mitwirkung der Glykosamino- 
glykano-Hydrolasen am physiologischen Mucopolysaccharid-Stoffwechsel des Tumors möglich, wobei die simultane oder successive Aktion von mindestens drei (Hyaluronatglykanohydrolase, $\beta$-Glucuronidase, $N$-Acetyl- $\beta$-glucosaminidase) wahrscheinlich jedoch mehr Glykosidasen notwendig ist. Hierfür spricht auch die Beobachtung, daß für die $\beta$-Glucuronidase die höchste Aktivität im mucopolysaccharidreichen Randgebiet des Tumors gefunden wurde (19) und daß neben $\operatorname{der} \beta$ Glucuronidase auch andere Glykosidasen im Tumorgewebe nachgewiesen wurden, wie ein die Viskosität von Hyaluronat reduzierendes Prinzip aus Rous-Sarkom (2) und ein Hyaluronat-hydrolysierendes Enzym sowie eine $\beta$-Glucosaminidase ${ }^{1}$ ) aus Asciteszellen (20). Zum anderen lassen die hohen im Walker-Carcino-Sarkom ge- fundenen Enzymaktivitäten daran denken, daß die enzymatische Hydrolyse der Mucopolysaccharide des benachbarten Bindegewebes des Wirtsorganismus das infiltrative expansive Wachstum des Tumors erleichtert, der Tumor also die ebenfalls mucopolysaccharidreiche bindegewebige Abwehrzone des Wirtsorganismus enzymatisch durchbricht.

Die Arbeit wurde durch die Deutsche Forschungsgemeinschaft und den Fonds der Chemischen Industrie in dankenswerter Weise unterstützt. Für die Durchführung eines Teils der enzymatischen Aktivitätsbestimmungen danken wir den Herren O. HoEfELE, G. ReICH und Dr. U. STEIN.

1) In Asciteszellhomogenaten fanden wir eine N-Acetyl- $\boldsymbol{\beta}$-glucosaminidase, die nach Reinigung durch Ammoniumsulfatfällung und Chromatographie in einer spezifischen Aktivität von $0,093 \mathrm{U} / \mathrm{mg}$ Protein erhalten werden konnte.

\title{
Literatur
}

1. Sylvén, B. Aminos ugarcontaining compounds in tumors, in: Balazs, E. und R. W. Jeanloz, The Amino sugars, Vol. II A, Academic Press, New York (1965). - 2. Caputo, A. und M. L. Marcante, Cancer Res. 19, 1010 (1959). ORR, S. F. D., Biochem. biophysica Acta (Amsterdam) 14, 173 (1954). - 3. WARREN, G. H., E. Horenstenn und J. Gray, Arch. Biochem. Biophysics 44, 107 (1953); Merker, H. J., H. Greiling und Th. Günther, Zschr. Krebsforsch. 63, 491 (1960). - 4. Rilex, J. E., The Mast cells, Livingstone, Edinburgh-London (1959). - 5. BUDDECKE, E., W. KröZ und E. Lanka, Hoppe-Seyler's Z. physiol. Chem. 331, 196 (1963); BUDdeCKE, E., Hoppe-Seyler's Z. physiol. Chem. 318, 33 (1960). - 6. Reissig, J. L., J. L. Strominger und L. F. Leloir, J. biol. Chemistry 193, 265 (1951). 7. Buddecke, E. und D. Platr, Hoppe-Seyler's Z. physiol. Chem. 343, 61 (1965). - 8. BUDDECKE, E. und E. Werries, Hoppe-Seyler's Z. physiol. Chem. 340, 257 (1965). - 9. Talalay, P., W. H. Fishman und C. Huggins, J. biol.
Chemistry, 166, 757 (1946). - 10. Anson, M. L., J. gen. Physiol. 22, 79 (1938). - 11. Frimmer, M. und E. Buddecke, Z. Naturforsch. 196, 789 (1964). - 12. GotrschaLK, A. und S. FAZEKAS DE ST. Groth, Biochim. biophysica Acta (Amsterdam) 43, 513 (1960). -13. Barker, S. A., M. StaceY und D. J. Tipper, Nature (London) 184, 68 (1959). - 14. Langley, O. K. und E. J. Ambrose, Nature (London) 204, 53 (1964). - 15. Matheson, A. T. und B. L. Tatrrie, Canad, J. Biochem. 42, 95 (1964). - 16. Jackson, D. S., D. B. Flickinger und J. E. Dunphy, Ann. N. Y. Acad. Sci. 86, 943 (1960). - 17. Lippman, M., Transact. N.Y. Acad. Sci. (Ser. II) 27, 342 (1965). - 18. Fishman, H. W. und A. J. Anlyan, Cancer. Res. 7, 808 (1947). - Fisher, E. R. und B. Fisher, Cancer. Res. 21, 527 (1961). - 19. Fishman, W. H., G. W. Mitchell, P. R. F. Borges, K. T. Ladue und M. Hayashi, Cancer. Res. 16, 118 (1963). - 20. GüNTHER, Th., M. Wenzel und T. Eberhard, diese Z. 3, 76 (1965).
Professor Dr. E. Buddecke, Physiol.-Chem. Institut der Universität 74 Tübingen, Auf dem Schnarrenberg

\section{Beitrag zur Aufklärung des thermischen Verhaltens von Bence-Jones-Proteinen}

\author{
Von H. Hauser, A. Holasek und I. Pascher \\ Aius dem Institut für Physiologische Chemie der Universität Graz (Vorstand: Prof. Dr. A. Holasek)
}

(Eingegangen am 7. März 1966)

Reaktionskinetisch unterscheidet sich die Hitzefällung und Hitzedenaturierung des im Rahmen dieser Arbeit untersuchten Bence-Jones-Proteins („BJP-R“) nicht von der ànderer Eiweißkörper. Eine Dissoziation des BJP-R konnte bei einer Ionenstärke $\mu=0,1$ weder nach dem Erhitzen auf $90-100^{\circ}$ noch bei $\mathrm{pH}$-Werten zwischen 2 und 10 nachgewiesen werden. Bei geringerer Ionenstärke $(\mu=0,01)$ findet man bei $\mathrm{pH}$-Werten unter 4 und über 9 eine Abnahme der Sedimentationskonstanten und eine Verzögerung bei der Gelfiltration. Beide Erscheinungen könnten jedoch auch durch den Ladungseffekt ohne Dissoziation erklärt werden. Da auch beim Ovalbumin die Hitzelöslichkeit mit steigender Temperatur über $100^{\circ}$ zunimmt, muß eine Dissoziation der Bence-Jones-Proteine nicht die Voraussetzung für die Hitzelöslichkeit sein.

Bence-Jones protein (,BJP-R") does not differ from other proteins in its kinetics of heat precipitation and heat denaturation. At an ionic strength $\mu=0.1$, dissociation of BJP-R could not be detected either after heating to $90-100^{\circ}$, or at $\mathrm{pH}$ values between 2 and 10 . At lower ionic strength $(\mu=0.01)$, below $\mathrm{pH} 4$ and above $\mathrm{pH} 9$, there is a decrease in the sedimentation constant and a retardation in gel filtration. Both effects, however, could be explained by changes in electrical charge, without dissociation. Since the temperature solubility of ovalbumin also increases with temperature above $100^{\circ}$, dissociation of Bence-Jones proteins need not be a requirement for heat solubility. 